\title{
Die Revolution geht weiter
}

Immer mehr Patienten, die chronisch an Hepatitis C erkrankt sind, können auf Heilung hoffen. Seit die Europäische Arzneimittelbehöre im Januar 2014 das erste hochwirksame Medikament zur interferonfreien Therapie gegen die Lebererkrankung zugelassen hat, verzeichnen Mediziner auf diesem Gebiet eine rasante Entwicklung.

Mit den jüngst erfolgten Zulassungen für "Ledipasvir/Sofosbuvir" als Einzeltablette sowie „Paritaprevir/r plus Ombitasvir" und "Dasabuvir" hat die Deutsche Gesellschaft für Gastroenterologie, Verdauungs- und Stoffwechselkrankheiten (DGVS) nun bereits zum vierten Mal in kürzester Zeit ihre Therapieempfehlungen ergänzt. Das aktuelle „Addenum “ zur "S 3-Leitlinie Hepatitis $C^{\prime \prime}$ ist auf der Homepage der DGVS abrufbar.

"Mit jedem neuen Medikament wird das Spektrum der Behandlungsmöglichkeiten erweitert", sagt Prof. Dr. Thomas Berg, Klinik für Gastroenterologie und Hepatologie am Universitätsklinikum Leipzig.,IInzwischen ist bei mehr als $90 \%$ aller Hepatitis C-Patienten eine Heilung möglich“. Die jüngst zur Kombinationstherapie zugelassenen Präparate kommen für Patienten mit He- patitis C-Viren der Genotypen 1 oder 4 als eine von je vier Therapieoptionen in Frage. Eine positive Entwicklung zeichne sich zudem bezüglich der hohen und vielfach diskutierten Behandlungskosten von Hepatitis C-Patienten ab, so Berg. In Deutschland haben sich Hersteller und Spitzenverband der Gesetzlichen Krankenversicherung (GKV) mit Ablauf des ersten Jahres nach Zulassung nun erstmals auf niedrigere Kosten für ein Medikament verständigt.

Die Empfehlungen der DGVS im Internet: www.dgvs.de/leitlinien/therapieder-chronischen-hepatitis-c/

Wien klin Mag 2015 · 18:37 DOI 10.1007/s00740-015-0046-5 Online publiziert: 2. April 2015 (c) Springer-Verlag Wien 2015

\section{Die neuen Medikamente zur} Hepatitis C-Therapie:

- Sofosbuvir. Zulassung in Europa im Januar 2014. Für alle HCV-Genotypen ( 1 bis 6 ). Als Kombinationstherapie mit anderen Medikamenten.

- Simeprevir. Zulassung im Mai 2014. Für die HCV-Genotypien 1 und 4. Als Kombinationstherapie mit anderen Medikamenten.

- Daclatasvir. Zulassung im August 2014. Für HCV-Genotypen 1 bis 6. Als Kombinationstherapie mit anderen Medikamenten.

- Ledipasvir. Zulassung im Dezember 2014. Für HCV Genotypen 1,3,4 und 6. Nur als Fixkombination mit Sofosbuvir.

- Paritaprevir + Ombitasvir und Dasabuvir (als Kombinationstherapie). Zulassung Januar 2015. Für HCV Genotyp 1.
Quelle:

Presseaussendung

Deutsche Gesellschaft für Gastroenterologie,

Verdauungs- und Stoffwechselkrankheiten (DGVS) 